\title{
EDITORIAL
}

\section{Overlap Syndrome: A Double Trouble}

\author{
Mahismita Patro ${ }^{1}$, Dipti Gothi ${ }^{2}$ \\ Indian Journal of Sleep Medicine (2021): 10.5005/jp-journals-10069-0075
}

Obstructive sleep apnea (OSA) and chronic obstructive pulmonary disease (COPD) are among the most common diseases encountered by pulmonologists. A substantial number of patients have both OSA and COPD attributable to common pathophysiological mechanisms or by chance alone. The association is known as "the overlap syndrome," the term first coined by Flenley. ${ }^{1}$ The overlap syndrome is clinically distinct from OSA or COPD alone with its unique course and prognosis. It is a common disease affecting at least $1 \%$ of the adult population. ${ }^{2}$ The burden of overlap syndrome in India is also high based on limited studies. ${ }^{3,4}$

The prevalence of the overlap syndrome is influenced by various pathophysiological interactions between COPD and OSA. Age, obesity, and smoking are shared risk factors for both disorders. The COPD patients have varied clinical spectrums ranging from the predominant emphysema to the predominant chronic bronchitis phenotype. The predominant chronic bronchitis phenotype predisposes to the development of OSA attributable to the higher body mass index (BMI) and right heart failure in them. Peripheral fluid retention leading to rostral fluid shift during the sleep in cor pulmonale also predisposes to upper airway narrowing and thus OSA. ${ }^{5}$

The patients with overlap syndrome have a worse clinical course associated with increased morbidity and mortality compared to COPD or OSA. They suffer more frequent episodes of nocturnal oxygen desaturation, greater daytime hypoxemia, and hypercapnia than either disease alone. They have a higher frequency of COPD exacerbations, severe respiratory symptoms, and poor quality of life. These patients also tend to develop severe respiratory failure requiring noninvasive ventilator during the exacerbation. They are also more prone to developing cardiovascular complications as arrhythmias and pulmonary hypertension. ${ }^{5,6}$

The presence of pulmonary hypertension in chronic lung disease is associated with a worse functional status and worse prognosis. Both OSA and COPD are common causes of group-3 pulmonary hypertension. The overlap syndrome increases the risk of pulmonary hypertension and right heart failure many folds. The prior studies have estimated a prevalence of $\mathrm{PH}$ in overlap syndrome to be 36 to $86 \%$. This is roughly five to seven times as compared to OSA or COPD alone. ${ }^{6}$ The underlying mechanisms for higher prevalence of cardiovascular complications are poorly understood and are the area of further research. This might be related to the higher prevalence of nocturnal oxygen desaturation, daytime hypoxemia, and hypercapnia in overlap patients as compared to either disease alone. ${ }^{5}$

In this issue of this journal, the authors have studied the prevalence of pulmonary hypertension in overlap syndrome thereby adding significant evidence from India. They have evaluated 100 patients with OSA via pulmonary function testing and history for the presence of COPD. They support the earlier findings of higher prevalence of overlap syndrome among OSA, seen in

\footnotetext{
1,2Department of Pulmonary Sleep and Critical Care Medicine, ESIC Hospital and PGIMSR, New Delhi, India
}

Corresponding Author: Dipti Gothi, Department of Pulmonary Sleep and Critical Care Medicine, ESIC Hospital and PGIMSR, New Delhi, India, Phone: 9971550550, e-mail: diptigothi@gmail.com

How to cite this article: Patro M, Gothi D. Overlap Syndrome: A Double Trouble. J Sleep Med 2021;16(2):21-22.

Source of support: Nil

Conflict of interest: None

$60 \%$ of their patients. Pulmonary hypertension was seven times more frequent in overlap syndrome than OSA alone, i.e., $36.67 \%$ vs $5 \%$ in their study. Thus, all the patients with OSA and COPD need to be screened for the presence of overlap syndrome in order to recognize this double trouble.

The screening for COPD among OSA is straightforward and primarily based on the clinical history and spirometry. However, the evaluation of COPD patients for the presence of OSA is an area of active research. The well-recognized screening tools for OSA have not been validated in COPD although STOPBANG was shown to be the most accurate among them. The demographic data such as $B M I \geq 25 \mathrm{~kg} / \mathrm{m}^{2}$, thick neck, and the presence of cardiovascular comorbidities were found to be better predictors compared to the traditional symptoms of snoring or excessive daytime sleepiness for identifying OSA in COPD. ${ }^{5,6}$ The confirmatory diagnosis of OSA requires overnight polysomnography, but it is limited by cost and availability. Overnight monitoring with pulse oximetry to look for nocturnal oxygen desaturation can be used as a screening tool for OSA in centers having limited resources. The presence of saw-toothing on overnight oximetry points toward OSA. ${ }^{7}$

The identification of overlap syndrome has important clinical consequences since the treatment of OSA in COPD improves the overall clinical outcome of COPD patients. The positive airway pressure (PAP) therapy improves the walking capacity, exercise tolerance, and lung functions in overlap syndrome. There is a reduction in COPD exacerbations and survival benefits with PAP therapy. The mean pulmonary artery pressure also has been shown to reduce with the treatment of OSA in overlap syndrome. Also, the appropriate management of COPD improves the clinical symptoms and compliance to PAP therapy in overlap syndrome. The patients with predominant OSA can be well managed with standard continuous PAP, whereas when COPD prevails, bilevel PAP is preferable. ${ }^{8,9}$

In conclusion, the overlap syndrome has a distinct clinical course with a worse prognosis and requires different management. Owing to the improved clinical outcome with early intervention in these patients, it is important for the clinicians to identify the patients with 
this double trouble. There is a need for further research focusing on the cardiovascular association with the overlap syndrome and screening methods for overlap syndrome in COPD.

\section{References}

1. Flenley DC. Sleep in chronic obstructive lung disease. Clin Chest Med 1985;6(4):651-661. DOI: 10.1016/s0272-5231(21)00402-0.

2. McNicholas WT. Chronic obstructive pulmonary disease and obstructive sleep apnoea-the overlap syndrome. J Thorac Dis 2016;8(2):236-242. DOI: 10.3978/j.issn.2072-1439.2016.01.52.

3. Gothi D, Gupta SS, Kumar N, et al. Impact of overlap syndrome on severity of acute exacerbation of chronic obstructive pulmonary disease. Lung India 2015;32(6):578-583. DOI: 10.4103/09702113.168132.

4. Nattusami L, Hadda V, Khilnani GC, et al. Co-existing obstructive sleep apnea among patients with chronic obstructive pulmonary disease. Lung India 2021;38(1):12-17. DOI: 10.4103/lungindia. lungindia_169_20.

5. McNicholas WT. COPD-OSA overlap syndrome evolving evidence regarding epidemiology, clinical consequences, and management. Chest 2017;152(6):1318-1326. DOI: 10.1016/j.chest.2017.04.160.

6. Xiong $M, H u W$, Dong $M$, et al. The screening value of ESS, SACS, $B Q$, And $S B Q$ on obstructive sleep apnea in patients with chronic obstructive pulmonary Disease. Int J Chron Obstruct Pulmon Dis 2019;14:2497-2505. DOI: 10.2147/COPD.S223354.

7. Netzer $\mathrm{N}$, Eliasson A, Netzer $\mathrm{C}$, et al. Overnight pulse oximetry for sleep-disordered breathing in adults. Chest 2001;120(2):625-633. DOI: 10.1378/chest.120.2.625.

8. Singh $\mathrm{S}$, Kaur H, Sigh S, et al. The overlap syndrome. Cureus 2018;10(10):e345. DOI: 10.7759/cureus.3453.

9. de Miguel J, Cabello J, Sánchez-Alarcos JM, et al. Long-term effects of treatment with nasal continuous positive airway pressure on lung function in patients with overlap syndrome. Sleep Breath 2002;6(1):3-10. DOI: 10.1007/s11325-002-0003-6. 